\title{
Getting Moore from DNA sequencing
}

The rapid drop in cost and increase in scale of DNA sequencing is often compared to the trend seen in the semiconductor industry in the second half of the twentieth century, which was described by Moore's law. Aptly, the aim to continue this progress in DNA sequencing is likely to be advanced by a technology based on semiconductors. Rothberg and colleagues now demonstrate the performance and scalability of this method - known as ion sequencing

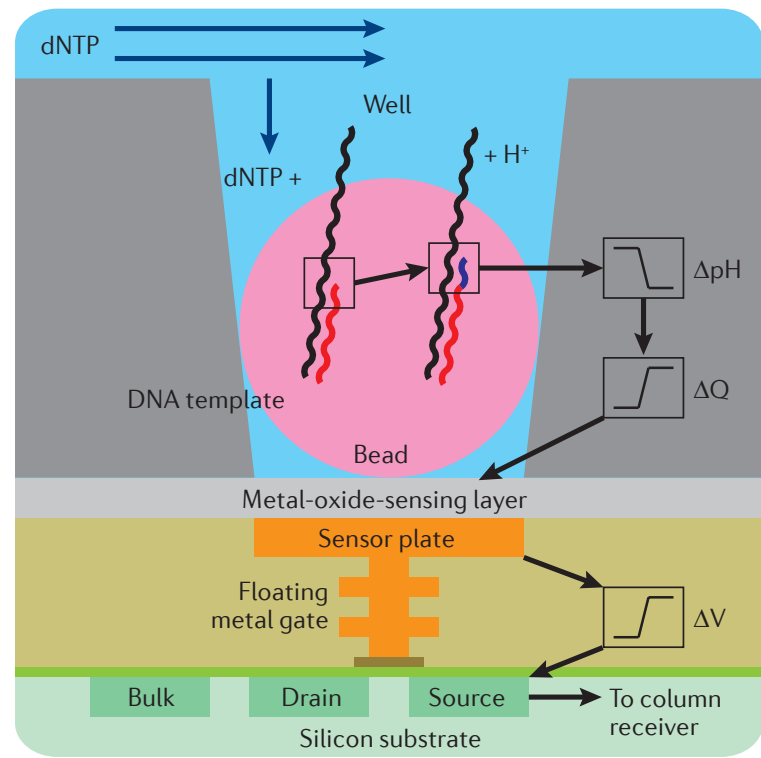

A simplified schematic diagram of an ion chip sensor well. Image is reproduced from Rothberg et al.
- on three bacterial genomes and one human genome.

In ion sequencing, fragments of genomic DNA are clonally amplified onto acrylamide beads. DNA polymerase and primers are then added to each bead-bound template strand, and the beads are delivered to individual wells on sequencing chips (see the figure). Each well contains a sensor that is highly sensitive to shifts in $\mathrm{pH}$ and converts the release of protons into a voltage. During a sequencing run, each of the four nucleotides is added in turn. When the DNA polymerase incorporates a nucleotide into the nascent DNA strand, a single proton is released, thus generating an electrical signal. The semiconductor electronics that connect the wells on the chip can rapidly gather and process the information from each well as the nucleotides are added, and the signals are then processed into base calls. This method is fast: an ion chip with 1.2 million sensors generates $\sim 25$ million bases in a 2 hour run. No modified nucleotides are required, which reduces cost compared with sequencing platforms that require fluorescently labelled nucleotides, for example.

The authors tested this technology on three bacterial genomes and routinely obtained read lengths of 100 bases or more. Comparison with the reference genomes of these species shows similar accuracy to sequencing using a light-based method for the first 50 bases of a read and higher accuracy over 100 bases. Rothberg et al. also demonstrate that this technology can be applied at a greater scale by sequencing the genome of Gordon Moore author of Moore's law - at a mean coverage of 10.6-fold. This required $\sim 1,000$ ion chips to provide 1 billion sensors, but the authors suggest that, in the future, 1 billion sensors could be provided on a single chip by harnessing the existing semiconductor technologies.

Ion sequencing, which was developed by Ion Torrent Systems, is one of several emerging so-called 'third-generation' sequencing technologies. Together with continued refinement of the second-generation platforms, genome sequencing looks set to continue to outstrip Moore's law.

Mary Muers

ORIGINAL RESEARCH PAPER Rothberg, J. M. et al. An integrated semiconductor device enabling non-optical genome sequencing. Nature 475, 348-352 (2011)

FURTHER READING Metzker, M. L. Sequencing technologies - the next generation. Nature Rev. Genet. 11, 31-46 (2010) 